\title{
Mapeamento da NBR ISO/IEC 12207 em Papéis da Gerência de Projetos de Software
}

\author{
Fábio Levy Siqueira, Paulo Sérgio Muniz Silva
}

\author{
Departamento de Engenharia de Computação e Sistemas Digitais - Escola Politécnica \\ da Universidade de São Paulo \\ Av. Prof. Luciano Gualberto, trav.3, n ${ }^{0} 158$ - 05508-900 - São Paulo - SP - Brazil \\ \{levy.siqueira, paulo.muniz\}@poli.usp.br
}

\begin{abstract}
The NBR ISO/IEC 12207 establishes a common framework for software life-cycle processes. One of these processes is the management, defined in the standard without the details of how to execute the prescribed activities. Seeking to create a set of practical guides of the software project management responsibilities, this paper presents a proposal for the NBR ISO/IEC 12207 mapping in a set of management roles based on the Rational Unified Process. One of the possibilities for this mapping is discussed in a case study, with distributed software development characteristics, where its use to the analysis of the software project management problems is presented.
\end{abstract}

Resumo. A NBR ISO/IEC 12207 define um quadro de referência padrão para os processos de ciclo de vida de software. Um desses processos é a gerência, definido na norma sem os detalhes de como executar as atividades prescritas. Buscando criar um conjunto de guias práticos das responsabilidades da gerência de projetos de software, nesse artigo é apresentada uma proposta de mapeamento da NBR ISO/IEC 12207 em um conjunto de papéis gerenciais baseados no Rational Unified Process. Uma das possibilidades desse mapeamento é discutida em um estudo de caso, com características de desenvolvimento distribuído de software, em que é apresentado o seu uso para a análise dos problemas da gerência de projetos de software.

\section{Introdução}

A gerência de projetos é um dos processos essenciais para qualquer projeto de desenvolvimento de software. Cabe ao gerente de projetos equilibrar adequadamente o custo, o prazo, a qualidade e o escopo do projeto para satisfazer as expectativas e as necessidades das partes envolvidas. Para realizar isso, o gerente deve executar atividades tais como desenvolver o cronograma, planejar os recursos, estimar o custo, desenvolver a equipe, identificar os riscos, entre diversas outras (Project Management Institute, 2000).

Uma definição para a gerência de projetos é apresentada no PMBOK - Project Management Body of Knowledge - (Project Management Institute, 2000), documento que busca identificar o conhecimento e prática normalmente aceitos para a gerência de projetos. Segundo o PMBOK, a gerência de projetos tem a seguinte definição:

"é a aplicação de conhecimentos, habilidades, ferramentas e técnicas para projetar atividades que atinjam requisitos de projeto" (Project Management Institute, 2000, p. 6). 
Para tanto, o gerente de projetos deve considerar as necessidades e as expectativas das partes envolvidas, contrabalançando-as com as demais considerações do projeto. Para que o gerente de projetos consiga corresponder às suas responsabilidades, é necessária a execução de algumas atividades que variam de importância dependendo das particularidades e da complexidade do projeto. Essas atividades podem ser observadas em alguns quadros de referência existentes na literatura que abordam de diversas formas e com diferentes enfoques as atividades gerenciais, como por exemplo, o já citado PMBOK, a NBR ISO/IEC 12207 (ABNT, 1998), o Capability Maturity Model Integration (CMMI) (CMMI Product Team, 2002) e a norma ISO/IEC 15504 (ISO, 1998). Entretanto o PMBOK não é específico ao desenvolvimento de software e as particularidades existentes em projetos desse tipo exigiriam uma extensão aos processos definidos. No caso da NBR ISO/IEC 12207, o processo gerencial é descrito com um alto nível de abstração e não cobre apenas a gerência de projetos. Por fim, o CMMI e a norma ISO/IEC 15504 não definem exatamente os processos gerenciais, tratando de boas práticas que tornem as atividades gerenciais mais adequadas para $\mathrm{o}$ desenvolvimento de software.

Buscando estudar as atividades da gerência de projetos de software com um enfoque prático, nesse artigo é apresentado um mapeamento do processo gerencial definido pela NBR ISO/IEC 12207 em um conjunto de papéis gerenciais baseados no Rational Unified Process (RUP) (2003). Esse mapeamento seguiu a filosofia definida pela norma, obtendo processos gerenciais para cada um dos papéis considerados. Usando esses processos como referência é possível, por exemplo, analisar os problemas ocorridos na gestão de um determinado projeto de software ou modelar processos condizentes com a norma para os papéis gerenciais.

Para apresentar esse mapeamento, o trabalho está estruturado da seguinte forma: na seção 2 é apresentada a NBR ISO/IEC 12207, enfatizando o processo de gerência e delimitando o escopo para o mapeamento. Na seção 3 é apresentada a divisão da gerência de projetos em papéis, a partir dos papéis gerenciais definidos no RUP. A partir dessa divisão e da NBR ISO/IEC 12207, na seção 4 é apresentado o método para realizar esse mapeamento. Na seção 5 é apresentado um estudo de caso em que se empregou esse mapeamento para a análise dos problemas da gerência de projetos, do ponto de vista do papel gerente de requisitos, com foco no desenvolvimento distribuído de software (desenvolvimento de software realizado por pessoas que não estão em proximidade física). Por fim, na seção 6 é apresentada a conclusão, abordando algumas considerações finais e perspectivas de trabalhos futuros.

\section{A NBR ISO/IEC 12207}

A NBR ISO/IEC 12207 define um quadro de referência padrão para os processos de ciclo de vida de software, entre eles a gerência, a serem implementados pelas organizações envolvidas com software (vide Figura 1). Por ser uma norma com um nível alto de abstração, não são especificados detalhes de como executar as atividades definidas, havendo liberdade para cada organização implantar a norma da forma que seja mais adequada às suas necessidades.

O processo gerencial definido na norma deve ser empregado especificamente para cada um dos processos fundamentais e de apoio, caso seja aplicável. Para o âmbito deste trabalho, o escopo da gerência definida pela NBR ISO/IEC 12207 foi limitado ao 
processo de desenvolvimento de software e aos processos de apoio. Dessa maneira, os processos organizacionais e os demais processos fundamentais foram considerados fora do escopo. Ademais, simplificaram-se as dependências do processo de desenvolvimento com os processos de apoio e organizacionais. Tal abstração permite a investigação das relações essenciais entre atividades da gerência no processo de desenvolvimento e papéis gerenciais.
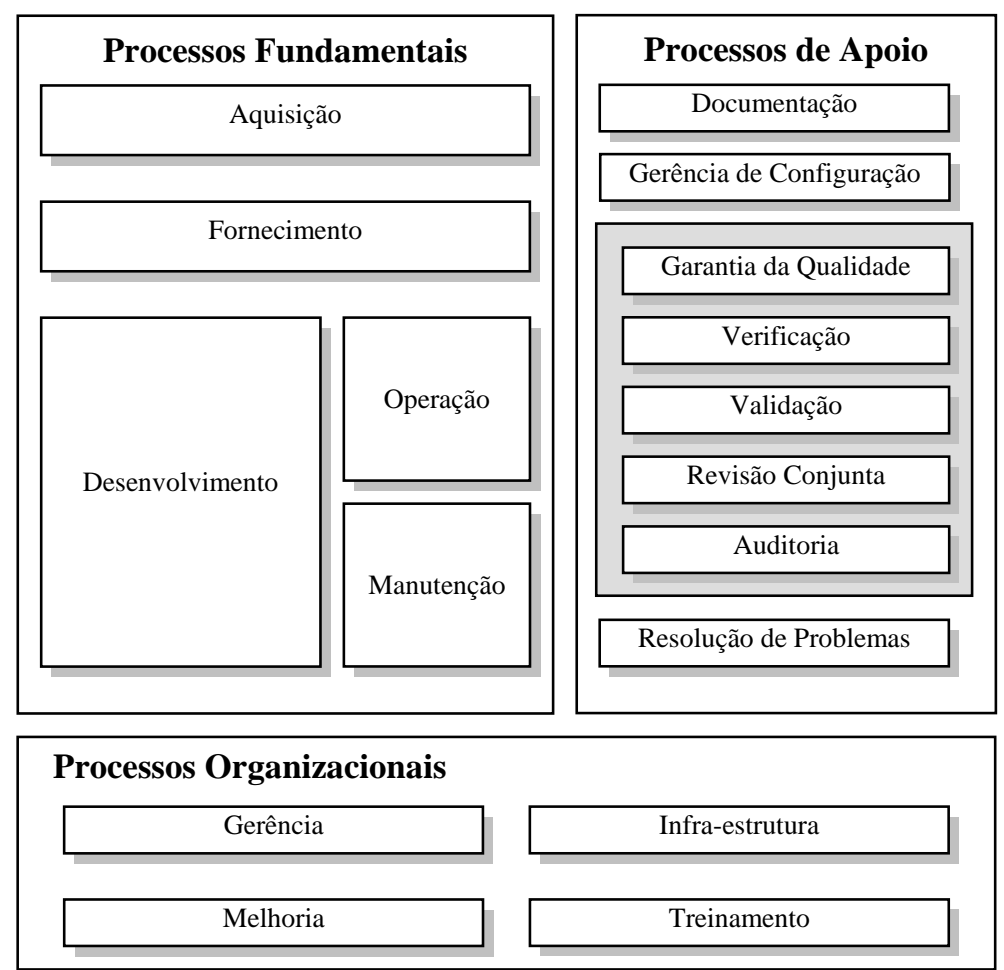

Figura 1: Os processos definidos pela norma NBR ISO/IEC 12207 (retirado de ABNT, 1998).

É importante ressaltar que a gerência de projetos é apenas uma parte da gerência definida na NBR ISO/IEC 12207. A abrangência da visão apresentada da gerência pela norma e o fato dela poder ser independente de projetos e contratos a torna mais geral que a gerência de projetos.

\subsection{O Processo Gerencial da NBR ISO/IEC 12207}

Considerando que o processo gerencial deve ser empregado por qualquer grupo que necessite ser gerenciado, são especificadas as seguintes atividades e tarefas para o processo gerencial como um todo (ABNT, 1998):

\section{- Iniciação e definição de escopo:}

$\circ$ definir os requisitos do processo;

- analisar se o processo é factível ao observar os recursos; e

- modificar os requisitos caso necessário e possível.

- Planejamento: preparar os planos para a execução do processo.

- Execução e controle:

○ iniciar a execução do plano observando os objetivos e critérios definidos; 


\section{Simpósio Brasileiro de Qualidade de Software}

- monitorar a execução do processo, relatando o progresso a quem for necessário;

- investigar, analisar e resolver problemas que ocorrerem durante a execução e, caso necessário, determinar, controlar e monitorar modificações nos planos; e

○ relatar o progresso do processo em pontos previamente definidos.

\section{- Revisão e avaliação:}

- garantir que os produtos de software e os planos estão sendo avaliados quanto a satisfação dos requisitos; e

○ julgar os resultados da avaliação dos produtos, atividades e tarefas completadas frente aos objetivos e ao plano.

\section{- Conclusão:}

- determinar se o processo está completo ao observar os critérios do contrato ou da organização após o término dos produtos, atividades e tarefas; e

- checar os resultados e registros dos produtos, atividades e tarefas observando sua completude.

\section{A Divisão da Gerência de Projetos em Papéis}

A gerência de projetos é responsável por diversas atividades dentro de um projeto, seja ao considerar a organização apresentada pela norma NBR ISO/IEC 12207 ou qualquer outro quadro de referência. Em projetos de pequeno porte essas atividades (ou melhor, as atividades gerenciais que forem cabíveis ao projeto) podem ser facilmente realizadas por uma única pessoa. Mas, conforme o projeto aumenta de tamanho, as atividades tornam-se mais complexas, muitas vezes necessitando a divisão do trabalho entre diversas pessoas. Para isso é necessária uma definição das responsabilidades de cada um dos gerentes de projeto para que não haja superposição de trabalho, ou pior que isso, a existência de atividades importantes que não sejam realizadas.

Um primeiro nível de divisão do trabalho gerencial pode ser realizado através da definição de um conjunto de papéis. De acordo com Kroll e Kruchten, um papel é:

"a definição do comportamento e responsabilidades de um indivíduo, ou um conjunto de indivíduos, trabalhando juntos como uma equipe” (Kroll e Kruchten, 2003, p.386).

Dessa forma, ao criar um conjunto de papéis gerenciais é possível dividir mais logicamente as diferentes atribuições existentes, diminuindo a complexidade individual de cada um dos papéis. Mas, para que um conjunto de papéis faça sentido, é necessário que ele esteja definido no contexto de uma metodologia (uma das dimensões de um processo $^{1}$ ). Sem saber exatamente como deve ser feito não é possível dividir as responsabilidades.

No quadro de referência definido pela NBR ISO/IEC 12207 não é definido um conjunto de papéis gerenciais. Mas, por ser um quadro de referência de processos, é até possível chegar-se a alguns papéis: uma divisão das responsabilidades gerenciais seria tratar a gerência para cada um dos processos considerados adequados para o projeto.

\footnotetext{
1 Uma visão das dimensões do processo é apresentada por Unhelkar (2003). Segundo o autor, um processo seria composto pela metodologia (como fazer), sociologia (quem faz) e tecnologia (o que fazer).
} 
Dessa forma, existiriam as gerências de desenvolvimento, de documentação, de configuração, de garantia da qualidade, de verificação, de validação, etc. Uma divisão desse tipo não parece muito adequada, uma vez que tal abordagem apresenta alguns pontos negativos do ponto de vista prático. De um lado, a gerência encarregada do processo de desenvolvimento seria responsável por um conjunto muito grande de atribuições. Do outro, alguns processos de apoio, que na prática são agrupados, seriam vistos como processos isolados. Considerando esses pontos negativos e também a arbitrariedade da escolha dessa divisão, parece ser mais apropriado determinar um conjunto de papéis a partir de algo mais prático, mais próximo da realidade diária.

$\mathrm{Na}$ procura por um bom conjunto de papéis gerenciais, uma divisão especialmente prática pode ser encontrada no Rational Unified Process (RUP). Como o RUP pode ser visto como um quadro de referência de processos de desenvolvimento de software, ele pode ser moldado para a aplicação em diversas situações, seja em um projeto de uma pessoa ou um projeto de grande porte (Kroll e Kruchten, 2003). Dessa forma, no RUP é apresentada uma boa diversidade de papéis gerenciais como segue (Rational Unified Process, 2003):

- Gerente de projetos: responsável por planejar, gerenciar, alocar recursos, definir prioridades e cuidar da equipe de projeto, entre outras responsabilidades.

- Gerente de controle de mudanças: responsável por definir e cuidar do controle de mudanças.

- Gerente de configuração: responsável pela infra-estrutura e ambiente da gerência de configuração.

- Gerente de testes: responsável pela atividade de testes.

- Gerente de implantação: responsável por planejar a transição do produto do ambiente de desenvolvimento para o ambiente real.

- Engenheiro de processos: responsável por moldar o processo mais adequado ao projeto e também outras tarefas relacionadas à educação e adequação do processo de desenvolvimento.

- Revisor de gerência: responsável por julgar o trabalho gerencial em pontos definidos do projeto.

Apesar da quantidade e diversidade de papéis, a gerência de projetos é coberta pelo RUP em apenas um subconjunto do que é importante para a sua perspectiva de desenvolvimento de software (Rational Unified Process, 2003). Ao usar o PMBOK como referência das responsabilidades da gerência de projetos, Charbonneau (2004) afirma que algumas áreas de conhecimento (gerência de custo, gerência de recursos humanos e gerência de aquisição) estão fora do escopo do RUP e não têm equivalentes entre as disciplinas definidas. Além disso, Charbonneau (2004) também aponta que o RUP não tem um mapeamento perfeito nas outras áreas de conhecimento do PMBOK, existindo diferenças nos documentos e nos processos (atividades, na nomenclatura do RUP) empregados. Entretanto, ao considerar os objetivos distintos do PMBOK e do RUP, percebe-se que essas diferenças são esperadas: enquanto o PMBOK busca descrever o conhecimento aceito da gerência de projetos, o RUP prescreve práticas para a gerência de projetos dentro do desenvolvimento de software (Charbonneau, 2004). Mesmo assim, não existem contradições significativas entre as práticas definidas pelos 
dois, o que permite dizer que o RUP é compatível com o PMBOK (Charbonneau, 2004).

Ainda considerando o mapeamento dos processos definidos pelo PMBOK com as atividades definidas pelo RUP (apresentado por Charbonneau (2004)), percebe-se que não são somente os papéis gerenciais colocados anteriormente que tratam da gerência de projetos no RUP. Um outro conjunto de papéis trata da gerência do escopo do projeto, materializada nas atividades que envolvem os requisitos do sistema:

- Analista de sistemas: responsável pela gerência de requisitos e a criação da visão do sistema.

- Especificador de requisitos: responsável por detalhar os requisitos do sistema.

- Revisor técnico: responsável pela revisão dos requisitos.

- Arquiteto: responsável pela priorização dos casos de uso.

Buscando criar um conjunto de papéis que representem adequadamente as responsabilidades da gerência de projetos, propõe-se aqui um papel gerencial que absorveria essas atribuições dispersas entre esses quatro papéis: o gerente de requisitos. Dessa maneira, na Figura 2 são apresentados cada um dos papéis gerenciais aqui considerados (os do RUP mais a proposição do papel de gerente de requisitos) e suas respectivas disciplinas do RUP.

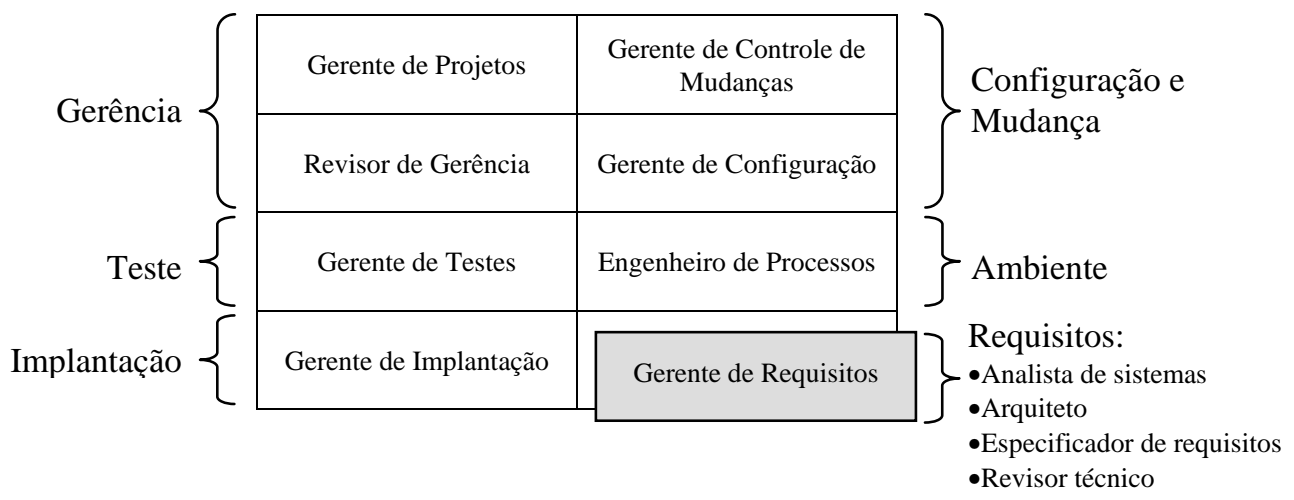

Figura 2: Os papéis gerenciais e suas respectivas disciplinas no RUP (2003) e, em evidência, o papel de gerente de requisitos e os papéis que ele incorpora.

É importante ressaltar que o RUP não cobre integralmente a ISO/IEC 12207. Kruchten (2002), ao considerar os papéis existentes e as disciplinas envolvidas no RUP, afirma que apenas alguns processos definidos pela ISO/IEC 12207 são cobertos pelo RUP (em sua versão 2002.05), seja de forma completa ou parcial:

- Completa: desenvolvimento, gerência de configuração, revisão conjunta, resolução de problemas e gerência.

- Parcial: manutenção, documentação, garantia da qualidade, verificação, validação, auditoria e melhoria.

Os demais processos teriam uma pequena cobertura ou então não seriam cobertos. Mesmo assim, Krutchen (2002) afirma que o RUP pode auxiliar a adoção da norma ISO/IEC 12207 devido à sua grande cobertura nos processos de desenvolvimento, de gerência e de apoio. 
Apesar das limitações do RUP em cobrir completamente a ISO/IEC 12207 e até mesmo o PMBOK, para o presente trabalho será utilizado como referência os papéis definidos pelo RUP, além do papel gerente de requisitos. Ao considerar a escolha dos processos mais adequados ao desenvolvimento de software em si (discutida na seção 2), percebe-se que o escopo do RUP é bastante semelhante ao escopo aqui definido. Com isso, apesar da limitação do RUP em cobrir a ISO/IEC 12207, os processos que não são por ele cobertos também não fazem parte do escopo deste trabalho, o que torna a divisão do RUP suficientemente adequada.

\section{A Divisão de Papéis e a Norma NBR ISO/IEC 12207}

Usando a NBR ISO/IEC 12207 como referência das atividades da gerência de projetos, cada um dos papéis definidos a partir do RUP é encarregado por parte das responsabilidades gerenciais. Um gerente de requisitos seria responsável apenas pela parte das atribuições da gerência de projetos que cuida da eliciação, organização e documentação dos requisitos, por exemplo.

Uma idéia para dividir as atividades para cada papel é instanciar o processo de gerência a partir do conjunto de atividades que sejam responsabilidade desse papel. No caso da gerência de requisitos, as atividades atreladas a esse papel seriam, entre outras, a Análise dos requisitos do sistema e a Análise dos requisitos do software, dentro do processo de desenvolvimento definido pela norma. A gerência para esse papel observaria essas atividades à luz do processo de gerência, instanciando uma gerência específica para essas atividades. O problema dessa abordagem é que a norma define o processo gerencial a partir do processo fundamental ou de apoio, e não para um conjunto qualquer de atividades. Com isso, deve-se observar a instanciação da gerência para um processo inteiro e separar as atividades e tarefas para um papel específico.

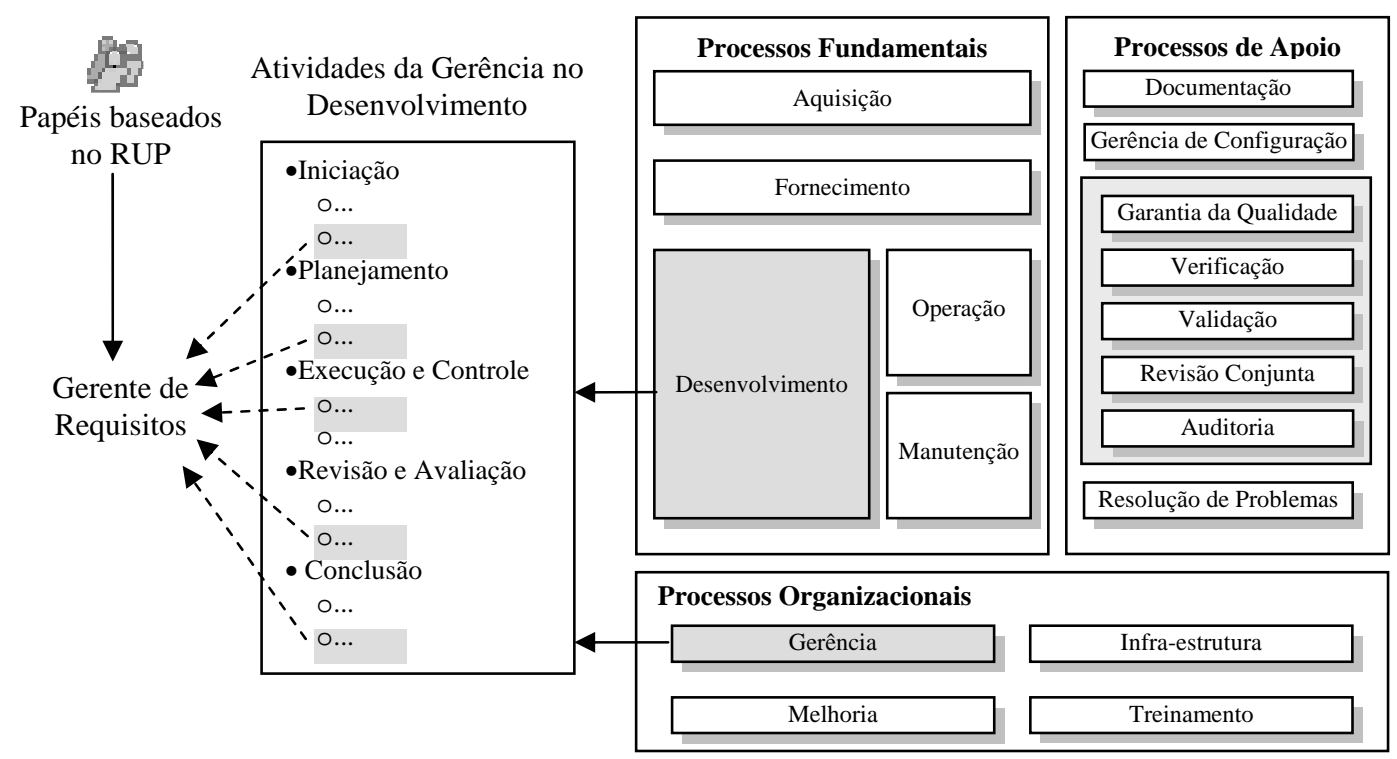

Figura 3: Exemplo da divisão das tarefas para cada um dos papéis gerenciais.

Usando como exemplo dessa abordagem o processo de desenvolvimento, a gerência, seguindo a norma deve considerar desde a análise dos requisitos do sistema, o design detalhado do software, a integração do sistema a até o apoio à aceitação. A partir dessas atividades de desenvolvimento são definidas as tarefas para a iniciação, 
planejamento, execução e controle, revisão e avaliação e conclusão, conforme apresentado na Figura 3. Ao considerar a divisão de papéis, algumas das tarefas instanciadas serão responsabilidades do gerente de requisitos. Outra parte será responsabilidade do gerente de testes e uma outra do gerente de integração, por exemplo.

Os papéis muitas vezes têm responsabilidades provenientes de mais de um processo. O engenheiro de processo, por exemplo, é responsável por parte da gerência do desenvolvimento e também por parte da gerência da documentação. Um mapeamento dos papéis baseados no RUP em relação à cobertura dos processos escolhidos da NBR ISO/IEC 12207 é apresentado na Tabela 1, fundamentado principalmente nas atividades definidas pela norma e pelos processos e objetivos de cada um dos papéis, de acordo com o RUP. Para criar o mapeamento foi analisado se as atividades e objetivos de cada papel estavam relacionados à gerência de cada tarefa definida pela norma. Como os papéis servem apenas como referência para agrupar um conjunto de responsabilidades, é importante notar que esse mapeamento extrapola o que é definido pelo RUP para que os papéis possam ser cobertos pela norma.

Tabela 1: Mapeamento dos papéis gerenciais e a NBR ISO/IEC 12207.

\begin{tabular}{|c|c|c|c|c|c|c|c|c|}
\hline Atividade & 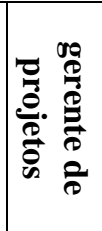 & 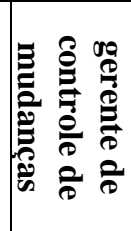 & 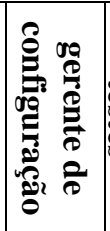 & 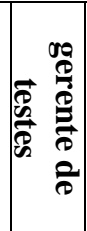 & 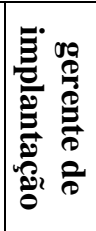 & 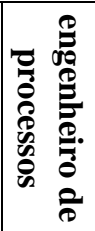 & 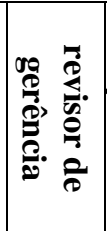 & 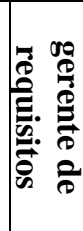 \\
\hline \multicolumn{9}{|l|}{ 5.3. Desenvolvimento } \\
\hline 5.3.1. Implementação do processo & & & & & & $\mathrm{X}$ & & \\
\hline 5.3.2. Análise dos requisitos do sistema & & & & & & & & $\mathrm{X}$ \\
\hline 5.3.3. Projeto da arquitetura do sistema & $\mathrm{X}$ & & & & & & & $\mathrm{X}$ \\
\hline 5.3.4. Análise dos requisitos do software & & & & & & & & $\mathrm{X}$ \\
\hline 5.3.5. Projeto da arquitetura do software & $\mathrm{X}$ & & & & & & & $\mathrm{X}$ \\
\hline 5.3.6. Projeto detalhado do software & $\mathrm{X}$ & & & $\mathrm{X}$ & & & & $\mathrm{X}$ \\
\hline 5.3.7. Codificação e testes do software & $\mathrm{X}$ & & & $\mathrm{X}$ & & & & $\mathrm{X}$ \\
\hline 5.3.8. Integração do software & $\mathrm{X}$ & & & $\mathrm{X}$ & & & & $\mathrm{X}$ \\
\hline 5.3.9. Teste de qualificação do software & $\mathrm{X}$ & & & $\mathrm{X}$ & & & & \\
\hline 5.3.10. Integração do sistema & $\mathrm{X}$ & & & $\mathrm{X}$ & & & & \\
\hline 5.3.11. Teste de qualificação do sistema & & & & $\mathrm{X}$ & & & & \\
\hline 5.3.12. Instalação do software & & & & & $\mathrm{X}$ & & & \\
\hline 5.3.13. Apoio à aceitação do software & & & & & $\mathrm{X}$ & & & \\
\hline \multicolumn{9}{|l|}{ 6.1. Documentação } \\
\hline 6.1.1. Implementação do processo & $\mathrm{X}$ & & & & & $\mathrm{X}$ & & \\
\hline 6.1.2. Projeto e desenvolvimento & & & & & & $\mathrm{X}$ & & \\
\hline 6.1.3. Produção & $\mathrm{X}$ & & $\mathrm{X}$ & & & & & \\
\hline 6.1.4. Manutenção & & & $\mathrm{X}$ & & & & & \\
\hline \multicolumn{9}{|l|}{ 6.2. Gerência de configuração } \\
\hline 6.2.1. Implementação do processo & & $\mathrm{X}$ & $\mathrm{X}$ & & & & & \\
\hline 6.2.2. Identificação de configuração & & & $\mathrm{X}$ & & & & & \\
\hline 6.2.3. Controle de configuração & & $\mathrm{X}$ & $\mathrm{X}$ & & & & & \\
\hline 6.2.4. Relato da situação da configuração & & $\mathrm{X}$ & $\mathrm{X}$ & & & & & \\
\hline 6.2.5. Avaliação da configuração & & & $\mathrm{X}$ & & & & & \\
\hline 6.2.6. Gerência de liberação e distribuição & & & $\mathrm{X}$ & & & & & \\
\hline \multicolumn{9}{|l|}{ 6.3. Garantia de qualidade } \\
\hline 6.3.1. Implementação do processo & $\mathrm{X}$ & & & & & & & \\
\hline
\end{tabular}




\begin{tabular}{|c|c|c|c|c|c|c|c|c|}
\hline Atividade & 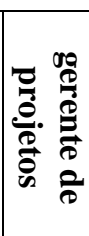 & 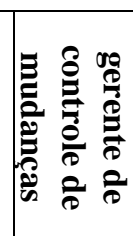 & 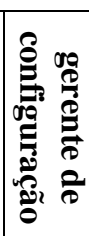 & 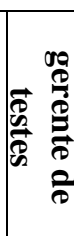 & 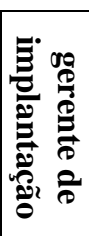 & 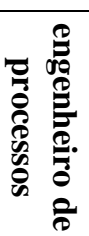 & 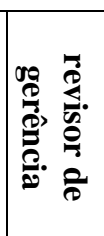 & 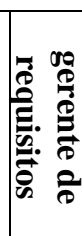 \\
\hline 6.3.2. Garantia do produto & $\mathrm{X}$ & & & & & & & \\
\hline 6.3.3. Garantia do processo & $\mathrm{X}$ & & & & & & & \\
\hline 6.3.4. Sistemas de garantia da qualidade & $\mathrm{X}$ & & & & & & & \\
\hline \multicolumn{9}{|l|}{ 6.4. Verificação } \\
\hline 6.4.1. Implementação do processo & $\mathrm{X}$ & $\mathrm{X}$ & $\mathrm{X}$ & $\mathrm{X}$ & $\mathrm{X}$ & $\mathrm{X}$ & $\mathrm{X}$ & $\mathrm{X}$ \\
\hline 6.4.2. Verificação & $\mathrm{X}$ & $\mathrm{X}$ & $\mathrm{X}$ & $\mathrm{X}$ & $\mathrm{X}$ & $\mathrm{X}$ & $\mathrm{X}$ & $\mathrm{X}$ \\
\hline \multicolumn{9}{|l|}{ 6.5. Validação } \\
\hline 6.5.1. Implementação do processo & $\mathrm{X}$ & & & & $\mathrm{X}$ & & $\mathrm{X}$ & \\
\hline 6.5.2. Validação & $\mathrm{X}$ & & & & $\mathrm{X}$ & & $\mathrm{X}$ & \\
\hline \multicolumn{9}{|l|}{ 6.6. Revisão Conjunta } \\
\hline 6.6.1. Implementação do processo & & & & & & & $\mathrm{X}$ & \\
\hline 6.6.2. Revisões de gerenciamento do projeto ${ }^{2}$ & $\mathrm{X}$ & $\mathrm{X}$ & $\mathrm{X}$ & $\mathrm{X}$ & $\mathrm{X}$ & $\mathrm{X}$ & $\mathrm{X}$ & $\mathrm{X}$ \\
\hline \multirow{2}{*}{\multicolumn{9}{|c|}{ 6.6.3. Revisões técnicas ${ }^{3}$}} \\
\hline & & & & & & & & \\
\hline 6.7.1. Implementação do processo & $\mathrm{X}$ & $\mathrm{X}$ & $\mathrm{X}$ & $\mathrm{X}$ & $\mathrm{X}$ & $\mathrm{X}$ & $\mathrm{X}$ & $\mathrm{X}$ \\
\hline 6.7.2. Auditoria & $\mathrm{X}$ & $\mathrm{X}$ & $\mathrm{X}$ & $\mathrm{X}$ & $\mathrm{X}$ & $\mathrm{X}$ & $\mathrm{X}$ & $\mathrm{X}$ \\
\hline \multicolumn{9}{|l|}{ 6.8. Resolução de Problemas ${ }^{5}$} \\
\hline 6.8.1. Implementação do processo & $\mathrm{X}$ & & & & & & & \\
\hline 6.8.2. Resolucão do problema & $\mathrm{X}$ & $\mathrm{X}$ & $\mathrm{X}$ & $\mathrm{X}$ & $\mathrm{X}$ & $\mathrm{X}$ & $\mathrm{X}$ & $\mathrm{X}$ \\
\hline
\end{tabular}

Como forma de avaliar o resultado obtido foi analisado o mapeamento feito com o existente na ISO/IEC TR 16326 (ISO, 1999). Essa norma é um guia para a aplicação da ISO/IEC 12207 para a gerência de projetos, contendo, por exemplo, um mapeamento do PMBOK (Project Management Institute, 2000) na gerência da norma ISO/IEC 12207 e também um mapeamento do processo de desenvolvimento no processo de gerência (ambos da norma ISO/IEC 12207), sendo que este último mapeamento foi o usado neste trabalho. Como o mapeamento aqui feito está contido no mapeamento proposto pela norma (a ISO/IEC TR 16326 mapeia, em alguns casos, as atividades e as tarefas de desenvolvimento em mais de uma atividade gerencial), pode-se dizer que a divisão é adequada. As diferenças que existem no resultado dos dois mapeamentos podem ser atribuídas a dois fatores principais:

1. a decisão de se simplificar a divisão ao mapear as tarefas à apenas uma atividade gerencial, e

2. o fato de o mapeamento proposto pela ISO/IEC 16326 não considerar papéis gerenciais e sim a gerência como um todo.

\footnotetext{
${ }^{2} \mathrm{O}$ revisor de gerência é encarregado dessa atividade, mas os demais gerentes também participam por serem alvos da revisão.

${ }^{3}$ Considerou-se que os gerentes não participam das revisões técnicas, apesar deles poderem participar do planejamento desse tipo de reunião.

${ }^{4} \mathrm{Na}$ norma, a auditoria é prescrita obrigatoriamente para o gerente de configuração. Mas os demais papéis podem também empregá-la.

${ }^{5} \mathrm{O}$ gerente de projetos é encarregado do plano de resolução de problemas mas, dependendo do problema, outro gerente pode ser encarregado de resolvê-lo.
} 
Os processos gerenciais sob a responsabilidade de um determinado papel são, a princípio, independentes. A ordem das atividades ao considerar todos os processos gerenciais será definida na instância do ciclo de vida considerado para um projeto particular. Com isso, enquanto se está realizando uma atividade de execução e controle para o processo de desenvolvimento, pode ser necessário, por exemplo, realizar por completo uma verificação (gerenciada pelo mesmo papel).

\section{Estudo de Caso}

A proposta apresentada anteriormente foi aplicada no projeto de desenvolvimento de um software de apoio para um sistema na área de astrofísica, organizado pelo Laboratório Nacional de Astrofísica (LNA), com o objetivo de analisar os problemas que ocorreram na gestão do projeto. O LNA é uma das unidades de pesquisa do Ministério de Ciência e Tecnologia e tem como missão principal fornecer meios e infraestrutura para que a comunidade astronômica brasileira possa realizar a sua pesquisa (Laboratório Nacional de Astrofísica, 2004). O projeto estudado foi a criação do software de redução de dados para um espectrógrafo de alta resolução (instrumento óptico responsável pela separação da radiação obtida por um telescópio).

Para criar esse software foi necessário realizar um desenvolvimento distribuído de software - desenvolvimento de software realizado por pessoas que não estão em proximidade física. Nesse tipo de desenvolvimento além dos problemas e dificuldades comuns a projetos em que as pessoas estão em proximidade física, existem inúmeros outros obstáculos para a realização das responsabilidades gerenciais, originários das características dessa forma de desenvolvimento (Siqueira e Muniz Silva, 2004). Como exemplo desses problemas e dificuldades, podem ser citados:

- a dificuldade de supervisionar e controlar o trabalho, pelo menos das formas tradicionais, ao considerar alguns agrupamentos (Harrington e Ruppel, 1999);

- a dificuldade de gerenciar conflitos ao considerar uma determinada distância física entre as partes envolvidas (Damian e Zowghi, 2003);

- a dificuldade de realização de reuniões gerenciais com pessoas chaves ao projeto dependendo do fuso-horário (Zowghi, 2002);

- os conflitos causados pelas diferentes culturas regionais (Herbsleb e Moitra, 2001);

- a dificuldade de comunicação entre as partes devido a diferenças de idioma (Mockus e Herbsleb, 2001);

- a necessidade de planejamento para a realização de viagens (passaporte, permissões de trabalho, etc) causadas por algumas diferenças dos locais (Kobitzch et al., 2001);

- as diferentes visões de qualidade dependendo das culturas organizacionais envolvidas (Kobitzch et al., 2001);

- a existência de diferentes estimativas devido à diferenças de infra-estrutura (Oppenheimer, 2002);

- a dificuldade de disseminação das informações do projeto devido a uma determinada relação de negócio (Kobitzch et al., 2001). 
Cabe à gerencia considerar esses e inúmeros outros obstáculos existentes devido às características do projeto, encontrando formas para superá-los ou gerenciá-los para que não tomem grandes dimensões.

Considerando as dificuldades dessa forma de desenvolvimento, o foco da análise dos problemas gerenciais concentrou-se nas dificuldades causadas pelo desenvolvimento distribuído de software. Para apresentar a análise realizada, o restante do artigo tratará apenas do papel gerente de requisitos, apesar de ter sido realizada a mesma análise para o papel engenheiro de processo. A escolha desses dois papéis para realizar a análise nesse estudo de caso foi baseada em uma observação inicial dos problemas mais importantes que ocorreram durante o projeto. Além disso, esses dois papéis são de extrema importância para o DDS. Ao considerar as diversas possibilidades de instanciação das características do DDS, cabe ao engenheiro de processos a responsabilidade de moldar um processo que seja otimizado às condições do projeto, buscando evitar as dificuldades e aproveitar dos benefícios. No caso da gerência de requisitos, mesmo em projetos tradicionais essa atribuição é essencial para o sucesso do projeto. Em uma pesquisa realizada por The Standish Group (1994) a realização adequada da gerência dos requisitos é um dos motivos mais citados para o sucesso de um projeto ${ }^{6}$. Em um DDS, a separação das partes envolvidas acaba tornando esse gerenciamento um desafio ainda maior.

\subsection{O Processo da Gestão do Desenvolvimento do Gerente de Requisitos}

Para analisar o projeto em questão foi criado o processo gerencial básico para o gerente de requisitos, seguindo o mapeamento aqui proposto (discutido na seção 4) da NBR ISO/IEC 12207 no conjunto de papéis baseados no RUP. Esse processo gerencial básico é apresentado a seguir (os números usados - ex: 5.3.1.3 - correspondem à identificação de tarefas definidas pela NBR ISO/IEC 12207 e em parênteses são apresentadas as tarefas gerenciais definidas pela norma que são tratadas pela tarefa proposta para o papel gerencial):

1. Iniciação

a. Estabelecer os requisitos do desenvolvimento em relação à gerência dos requisitos: selecionar os padrões, ferramentas, etc, para a execução da gerência de requisitos conforme a tarefa 5.3.1.3 (7.1.1.1).

b. Estabelecer a viabilidade do desenvolvimento em relação à gerência de requisitos (7.1.1.2).

c. Modificar os requisitos da gerência de requisitos conforme necessário e possível (7.1.1.3).

2. Planejamento

a. Planejar a gerência dos requisitos conforme a tarefa 5.3.1.4 (7.1.2.1).

3. Execução e controle

a. Executar os planos (7.1.3.1).

b. Realizar a gerência de requisitos através das atividades 5.3 .2 e 5.3 .4 (7.1.3.1).

\footnotetext{
${ }^{6}$ Para essa conclusão foram considerados os fatores envolvimento do usuário, requisitos claros e expectativas realísticas como responsabilidades da gerência dos requisitos.
} 
c. Realização da gerência de requisitos ao gerenciar as tarefas 5.3.3.2, 5.3.5.2, 5.3.6.7, 5.3.7.5 e 5.3.8.5 (7.1.3.1).

d. Monitorar a execução do processo e informar as partes (7.1.3.2).

e. O gerente deve investigar, analisar e resolver os problemas descobertos durante a execução do processo, o que pode resultar em mudanças dos planos (devendo documentar tudo), conforme a tarefa 5.3.1.2 (7.1.3.3).

f. Relatar o andamento do desenvolvimento em relação aos requisitos, declarando aderência aos planos (7.1.3.4).

4. Revisão e avaliação

a. Garantir que os resultados e os produtos são avaliados para a satisfação dos requisitos (7.1.4.1).

b. Julgar os resultados da avaliação dos produtos, atividades e tarefas para a satisfação dos objetivos e completude dos planos (7.1.4.2).

5. Conclusão

a. Determinar o fim da gerência dos requisitos (após as atividades, tarefas e produtos estarem completos) observando os critérios definidos em contrato ou procedimento da organização (7.1.5.1).

b. Checar os resultados e registros por completude, posteriormente os arquivando (7.1.5.2).

\subsection{Análise dos Problemas das Atividades da Gerência de Requisitos}

Para a análise dos problemas de gestão, especialmente os referentes à gerência de requisitos, foi elaborado um questionário cujas questões abordavam como cada uma das atividades do processo gerencial básico foi realizada, com o foco nas dificuldades causadas pelo desenvolvimento distribuído de software. Além disso, o questionário criado também obteve outras informações, tentando entender o contexto do projeto e o motivo das atividades serem realizadas da maneira que foram.

A análise das repostas permitiu observar que as atividades da gerência de requisitos foram realizadas de modo informal, sempre buscando obter um consenso de todas as partes envolvidas (o que poderia demorar devido à distribuição das pessoas):

- A iniciação foi executada rapidamente, mas mesmo assim o único problema existente foi a falta de análise dos recursos disponíveis para viagens.

- O planejamento foi executado informalmente com todas as partes envolvidas.

- A atividade de execução e controle foi realizada com o envolvimento de todas as partes envolvidas. Existiram diversos problemas, mas a maioria deles foi de natureza técnica. Os principais problemas causados pelo desenvolvimento distribuído de software foram a impossibilidade de participação de algumas pessoas nas reuniões e a dificuldade de discutir à distância.

- A revisão e avaliação foram executadas por todos os envolvidos, usando basicamente o e-mail para discutir os resultados (o que foi demorado).

- A conclusão também foi feita com o consenso das partes envolvidas.

Usando as informações obtidas com a análise dos problemas da gerência de requisitos e diversos problemas discutidos em outros relatos de experiência existentes na literatura, 
discutiu-se algumas recomendações para facilitar a realização das suas responsabilidades em um desenvolvimento distribuído de software. Como exemplo dessas recomendações, pode-se citar:

- considerar a necessidade de infra-estrutura de comunicação para a realização adequada das atividades (da tarefa de iniciação estabelecer os requisitos do desenvolvimento em relação à gerência dos requisitos);

- Analisar a possibilidade de uso das ferramentas idealizadas devido à infraestrutura do local. (da tarefa de iniciação estabelecer a viabilidade do desenvolvimento em relação à gerência de requisitos).

- considerar a necessidade da realização de reuniões em proximidade física (da tarefa de planejamento plano da gerência de requisitos); e

- considerar a maior dificuldade de negociação dos requisitos à distância (da tarefa de execução e controle realização da gerência de requisitos).

\section{Conclusão}

A gerência de projetos é encarregada de diversas responsabilidades dentro de um projeto. Cabe ao gerente de projetos planejar (nas diversas vistas existentes em um projeto), controlar e monitorar o andamento do que foi planejado e também motivar a equipe de desenvolvimento

Apesar de a NBR ISO/IEC 12207 tratar da gerência de projetos, o fato dessa norma trabalhar em um alto nível de abstração dificulta a aplicação do processo gerencial definido de uma maneira mais prática. Tratando dessa dificuldade, com o foco no desenvolvimento de software, nesse artigo foram apresentados um método e o embasamento empregados para mapear o processo gerencial da NBR ISO/IEC 12207 em um conjunto de papéis baseados no RUP, estendido com o papel de gerente de requisitos, criando assim processos gerenciais para cada um desses papéis. A aplicação desse mapeamento para a análise do processo gerencial realizado em um projeto foi também discutida nesse artigo através de um estudo de caso, enfatizando o processo gerencial básico para o gerente de requisitos.

Em trabalhos futuros pretende-se definir os processos gerenciais dos demais papéis (por enquanto foram tratados somente os papéis gerente de requisitos e engenheiro de processo). Além disso, pretende-se também utilizar esse mapeamento como referência para a melhoria do processo empregado no estudo de caso realizado (uma vez que será realizado um projeto semelhante a esse), buscando deixá-lo em conformidade com a NBR ISO/IEC 12207.

\section{Agradecimentos}

Essa pesquisa foi financiada por uma bolsa de mestrado fornecida pela Escola Politécnica da Universidade de São Paulo e pela CNPq.

\section{Referências}

ABNT - Associação Brasileira de Normas Técnicas. NBR ISO/IEC 12207 - tecnologia de informação: processos de ciclo de vida de software. ABNT, 1998. 
CHARBONNEAU, S. Software Project Management - A Mapping Between RUP and the PMBOK. The Rational Edge, May 2004. Disponível em: <http://www.therationaledge.com>.

CMMI PRODUCT TEAM. CMMI for Software Engineering, Version 1.1, Continuous Representation. Report CMU/SEI-2002-TR-028. Carnegie Mellon Software Engineering Institute, 2002. Disponível em: 〈http://www.sei.cmu.edu>.

DAMIAN, D.; ZOWGHI, D. An insight into the interplay between culture, conflict and distance in globally distributed requirements negotiations. In: HAWAII INTERNATIONAL CONFERENCE ON SYSTEM SCIENCES, 36., Hawaii, 2003.

HARRINGTON, S. J.; RUPPEL, C. P. Telecommuting: A Test of Trust, Competing Values, and Relative Advantage. IEEE Transactions on Professional Communication, v.42, n.4, p.223-239, December 1999.

HERBSLEB, J.; MOITRA, D. Global Software Development. IEEE Software, v.18, n.2, p.16-20, March/April 2001.

ISO - International Organization For Standardization. ISO/IEC TR 15504-1 - Software process assessment - Part 1: Concepts and introductory guide. ISO, 1998.

ISO - International Organization For Standardization. ISO/IEC TR 16326 - Software engineering -- Guide for the application of ISO/IEC 12207 to project management. ISO, 1999.

KOBITZSCH, W.; ROMBACH, D.; FELDMANN, R. L. Outsourcing in India. IEEE Software, v.18, n.2, p.78-86, March/April 2001.

KROLL, P.; KRUCHTEN, P. The Rational Unified Process Made Easy: a Practitioner's Guide to the RUP. Addison-Wesley, 2003.

KRUCHTEN, P. How the Rational Unified Process Supports ISO 12207. The Rational Edge. August 2002. Disponível em: 〈http://www.therationaledge.com〉.

LABORATÓRIO NACIONAL DE ASTROFÍSICA. Sobre o LNA. 2004. Informações sobre o LNA, seu histórico e sua organização. Disponível em: <http://www.lna.br/lna/lna_hist.html>.

MOCKUS, A.; HERBSLEB, J. D. Challenges of Global Software Development. In: INTERNATIONAL SOFTWARE METRICS SYMPOSIUM, 7., London, 2001.

OPPENHEIMER, H. L. Project Management Issues in Globally Distributed Development. In: INTERNATIONAL WORKSHOP ON GLOBAL SOFTWARE DEVELOPMENT, Orlando, 2002.

PROJECT MANAGEMENT INSTITUTE. A guide to the project management body of knowledge (PMBOK guide). Project Management Institute, 2000.

RATIONAL UNIFIED PROCESS, version 2003.06.12.01. Rational Software Corporation, 2003. Disponível para avaliação em: <http://www.rational.com>.

SIQUEIRA, F. L.; MUNIZ SILVA, P. S. As Características do Desenvolvimento Distribuído de Software. In: SIMPÓSIO BRASILEIRO DE SISTEMAS DE INFORMAÇÃO, Porto Alegre, 2004, p.171-178. 
THE STANDISH GROUP. The Chaos Report. 1994. Disponível em: $<$ http://www.standishgroup.com>.

UNHELKAR, B. Process Quality Assurance for UML-Based Projects. AddisonWesley, 2003.

ZOWGHI, D. Does Global Software Development Need a Different Requirements Engineering Process? In: INTERNATIONAL WORKSHOP ON GLOBAL SOFTWARE DEVELOPMENT, Orlando, 2002. 